\title{
University of Florida Potato Variety Spotlight: Red LaSoda'
}

\author{
Lincoln Zotarelli, Doug Gergela, Chad M. Hutchinson, David Dinkins, and Edsel Redden²
}

General Comments: Red LaSoda is the red-skinned fresh-market potato standard for Florida. Red LaSoda was observed in 1949 as a deep red mutant of LaSoda in the Louisiana potato breeding program. Originally, LaSoda was a selection from a cross of Triumph and Katahdin. Red LaSoda was released by the USDA and the Louisiana Agricultural Experiment Station in 1953. It has been in trials over many seasons and at many locations in Florida including university and grower sites. Production and quality results provided here are summarized from the redskinned fresh-market trials conducted by the University of Florida over the past 14 seasons.

General Characteristics: Red LaSoda has white to cream-colored flesh with a round to oblong tuber shape. It has smooth, red to pink-colored skin with deep to intermediate eye depth. The variety has good yield potential and a relatively low specific gravity compared to other red-skinned potato varieties adapted for Florida production (Tables 1 and 2).

Season Length: The time from planting to vine kill is approximately 85-95 days depending on growing conditions during the season. Tuber size should be checked regularly late in the season. Plants should be vine killed when size distribution meets desired goals. The time from vine kill to harvest varies by season. At least two to three weeks should be allowed for tubers to mature and set skin.

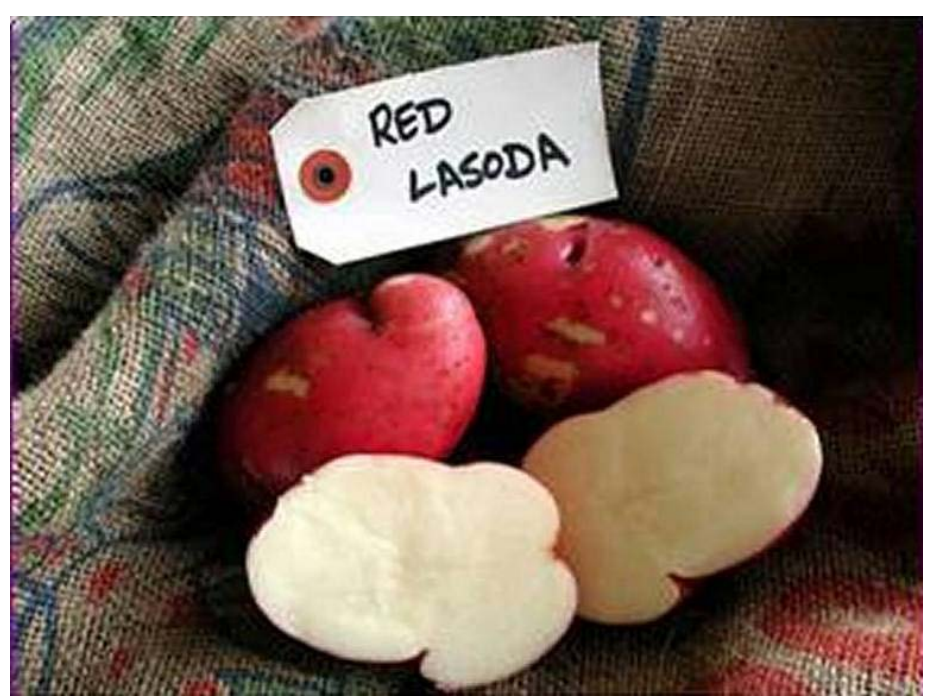

Figure 1. Typical tuber skin and internal flesh color of Red LaSoda. Credits: C. Hutchinson

Fertilization and Growth: University trials have used approximately $200 \mathrm{lb} \mathrm{N} /$ acre. Nitrogen should be managed so that it "runs out" late in the season to improve tuber maturity and skin set. It may require less nitrogen than the UF/IFAS Extension general recommendation of $200 \mathrm{lb} \mathrm{N} /$ acre in heavier soils to achieve this goal. $\mathrm{P}$ and $\mathrm{K}$ should be applied based on soil tests.

Foliage develops rapidly with determinant growth resulting in early to mid-season maturity.

1. This document is HS1078, one of a series of the Horticultural Sciences Department, UF/IFAS Extension. Original publication date July 2006. Revised August 2013. Visit the EDIS website at http://edis.ifas.ufl.edu.

2. Lincoln Zotarelli, Doug Gergela, and Chad Hutchinson, Horticultural Sciences Department, University of Florida/IFAS; David Dinkins, St. Johns County Cooperative Extension Office; Edsel Redden, Putnam County Cooperative Extension Office, UF/IFAS Extension, Gainesville, 32611. 
Planting: Tubers should break dormancy before planting. A seed piece of $2 \frac{1}{2}$ to $3 \mathrm{oz}$ is desired for planting. Plant spacing should be 5 to 8 inches in-row with 36 to 40 inches between rows. Closer in-row spacing will reduce harvested tuber size. Excessive soil moisture late in the season will degrade lenticel appearance and delay skin set.

Diseases: Red LaSoda has general disease resistance characteristics. The variety is susceptible to scab, early blight, late blight, corky ring spot, and bacterial wilt. A standard extension-recommended disease control program should be followed.

Seed Source: Open variety, available through many seed sources.

Other Information: Hutchinson, C. M., E. H. Simonne, G. J. Hochmuth, D. N. Maynard, W. M. Stall, S. M. Olson, S. E. Webb, T. G. Taylor and S. A. Smith. 2006. "Potato Production in Florida." In: Vegetable Production Guide for Florida. S. M. Olson and E. H. Simonne, eds. University of Florida.

Potato Association of America variety database: http:// potatoassociation.org/Industry\%20Outreach/varieties/ Red\%20Rounds/red_la_soda.html 
Table 1. Summary of production statistics and specific gravity of Red LaSoda, a red-skinned fresh-market potato variety grown at the UF/IFAS research and demonstration farm in Hastings, FL

\begin{tabular}{|c|c|c|c|c|c|c|c|c|c|c|c|c|}
\hline \multirow[t]{3}{*}{ Year } & \multirow{3}{*}{$\begin{array}{l}\begin{array}{l}\text { Total } \\
\text { Yield }\end{array} \\
\text { (cwt/A) }\end{array}$} & \multirow{3}{*}{$\begin{array}{c}\begin{array}{c}\text { Marketable } \\
\text { Yield' }\end{array} \\
\text { (cwt/A) }\end{array}$} & & & & \multicolumn{3}{|c|}{ Size } & & & \multirow{2}{*}{$\begin{array}{c}\text { Size } \\
\text { Class } \\
\%\end{array}$} & \multirow[b]{3}{*}{$\begin{array}{l}\text { Specific } \\
\text { Gravity }\end{array}$} \\
\hline & & & \multicolumn{6}{|c|}{ Distribution by Class $\%^{2}$} & \multicolumn{2}{|c|}{ Range \% } & & \\
\hline & & & C & B & A1 & A2 & A3 & A4 & $A 1$ to $A 3$ & A2 to $A 4$ & Culls & \\
\hline 1998 & 469 & 352 & -- & 3 & 23 & 35 & 18 & 0 & 76 & 53 & 22 & 1.058 \\
\hline 1999 & 396 & 329 & -- & 4 & 64 & 18 & 1 & 0 & 83 & 19 & 13 & 1.053 \\
\hline 2000 & 326 & 298 & -- & 4 & 35 & 38 & 18 & 0 & 91 & 46 & 5 & 1.072 \\
\hline 2001 & 305 & 279 & -- & 3 & 42 & 42 & 13 & 0 & 97 & 55 & 6 & 1.064 \\
\hline 2002 & 373 & 341 & -- & 3 & 43 & 44 & 10 & 0 & 97 & 54 & 6 & 1.061 \\
\hline 2003 & 495 & 419 & -- & 3 & 37 & 34 & 20 & 1 & 92 & 55 & 7 & 1.059 \\
\hline 2004 & 402 & 326 & 7 & 6 & 55 & 26 & 6 & 0 & 88 & 32 & 7 & 1.070 \\
\hline 2005 & 337 & 299 & 1 & 7 & 55 & 34 & 3 & 0 & 92 & 37 & 3 & 1.066 \\
\hline 2006 & 431 & 399 & 1 & 4 & 66 & 28 & 0 & 0 & 95 & 28 & 3 & 1.063 \\
\hline 2007 & 377 & 329 & 1 & 10 & 64 & 20 & 60 & 00 & 89 & 26 & 2 & 1.060 \\
\hline 2008 & 276 & 192 & 3 & 5 & 64 & 5 & 2 & 0 & 71 & 7 & 3 & 1.068 \\
\hline 2009 & 435 & 338 & 1 & 6 & 50 & 20 & 23 & 0 & 93 & 43 & 16 & 1.058 \\
\hline 2010 & 375 & 294 & 2 & 12 & 73 & 8 & 5 & 0 & 86 & 10 & 73 & 1.076 \\
\hline 2011 & 383 & 262 & 3 & 12 & 55 & 23 & 7 & 0 & 84 & 22 & 55 & 1.058 \\
\hline 2012 & 282 & 138 & 2 & 7 & 47 & 12 & 31 & 0 & 90 & 48 & 47 & 1.054 \\
\hline Average & 377 & 306 & 2 & 6 & 52 & 26 & 11 & 0 & 88 & 36 & 18 & 1.063 \\
\hline
\end{tabular}

'Marketable yield: Sum of size classes A1 to A3.

${ }^{2}$ Size classes: $C=1 / 2$ to $1 \frac{1}{2}$ inches, $B=1 \frac{1}{2}$ to $17 / 8$ inches, $A 1=17 / 8$ to $2 \frac{1}{2}$ inches, $A 2=2 \frac{1}{2}$ to $3 \frac{1}{4}$ inches, $A 3=3 \frac{1}{4}$ to 4 inches, $A 4>4$ inches;

Size distribution by class: Class (wt)/(Total Yield [wt] - culls [wt])

Table 2. Yield, vine maturity, tuber characteristics, and internal tuber defects of Red LaSoda, a red-skinned fresh-market potato variety grown at the UF/IFAS research and demonstration farm in Hastings, FL

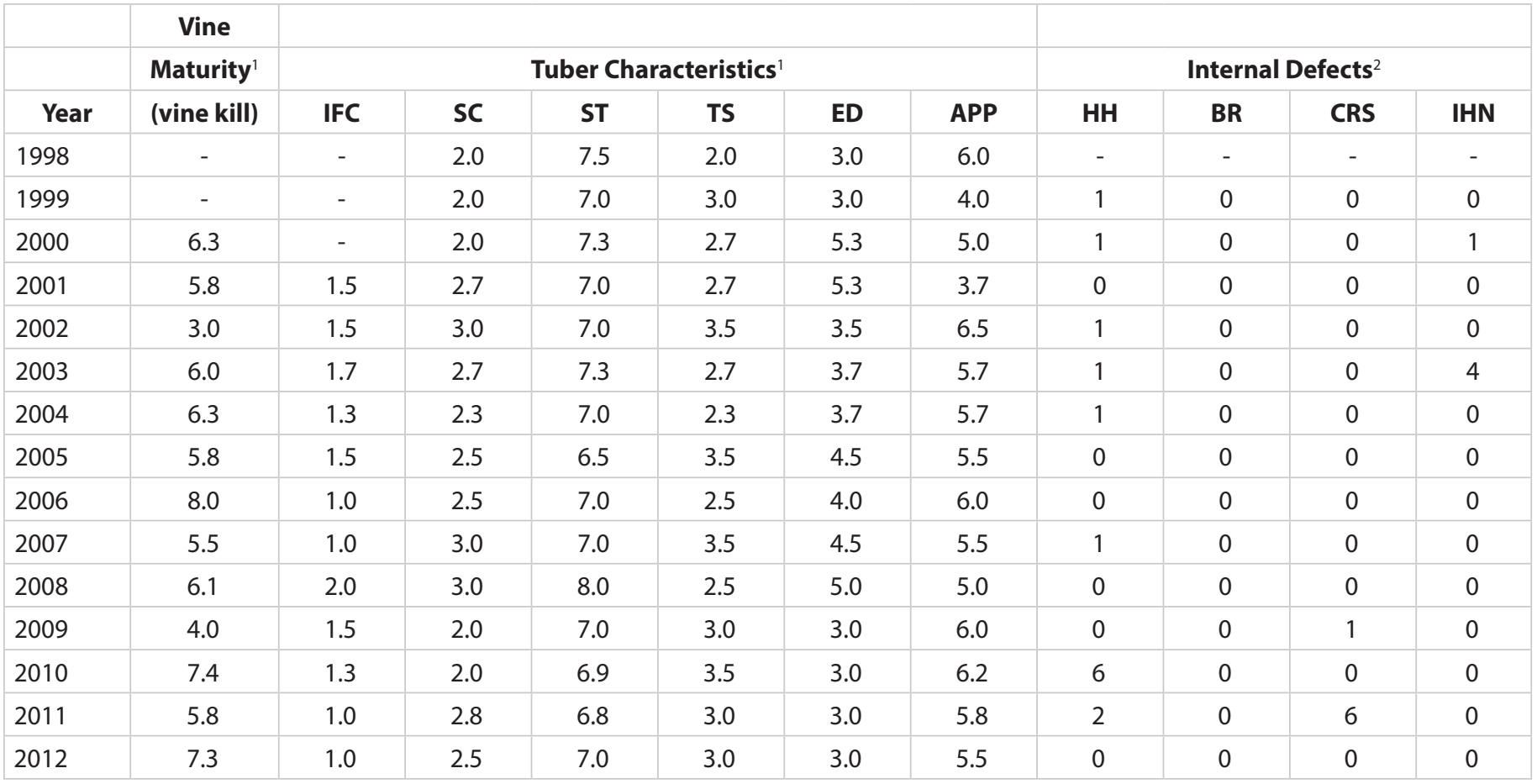

${ }^{1}$ See rating system outlined in Florida Rating Code Table (Table 3).

${ }^{2}$ Percent tuber defects. $\mathrm{HH}=$ hollow heart, $\mathrm{BR}=$ brown rot, $\mathrm{CRS}=$ corky ring spot, $\mathrm{IHN}=$ internal heat necrosis. 
Table 3. Florida rating codes for potato tuber characteristics ${ }^{1}$

\begin{tabular}{|c|c|c|c|c|c|c|c|}
\hline \multicolumn{8}{|c|}{ Tuber Characteristics } \\
\hline $\begin{array}{l}\text { Rating } \\
\text { Code }\end{array}$ & $\begin{array}{c}\text { Vine } \\
\text { Maturity }\end{array}$ & $\begin{array}{c}\text { Internal } \\
\text { Flesh Color }\end{array}$ & $\begin{array}{l}\text { Skin } \\
\text { Color }\end{array}$ & $\begin{array}{c}\text { Skin } \\
\text { Texture }\end{array}$ & $\begin{array}{l}\text { Tuber } \\
\text { Shape }\end{array}$ & $\begin{array}{c}\text { Eye } \\
\text { Depth }\end{array}$ & $\begin{array}{c}\text { Overall } \\
\text { Appearance }\end{array}$ \\
\hline 1 & dead & white & purple & partial russet & round & very deep & very poor \\
\hline 2 & +- & cream & red & heavy russet & mostly round & -- & -- \\
\hline 3 & yellow and dying & light yellow & pink & moderate russet & $\begin{array}{l}\text { round to } \\
\text { oblong }\end{array}$ & deep & poor \\
\hline 4 & +- & medium yellow & dark brown & light russet & mostly oblong & -- & -- \\
\hline 5 & $\begin{array}{c}\text { moderately } \\
\text { senesced }\end{array}$ & dark yellow & brown & netted & oblong & intermediate & fair \\
\hline 6 & +- & pink & $\tan$ & slightly netted & oblong to long & -- & -- \\
\hline 7 & $\begin{array}{l}\text { starting to } \\
\text { senesce }\end{array}$ & red & buff & $\begin{array}{l}\text { moderately } \\
\text { smooth }\end{array}$ & mostly long & shallow & good \\
\hline 8 & +- & blue & white & smooth & long & -- & -- \\
\hline 9 & $\begin{array}{l}\text { green and } \\
\text { vigorous }\end{array}$ & purple & cream & very smooth & cylindrical & very shallow & excellent \\
\hline
\end{tabular}

'Based on the standard NE 1031 rating codes for plant and tuber characteristics. 